\title{
Model of the Phase Transition Mimicking the Pasta Phase in Cold and Dense Quark-Hadron Matter
}

\author{
Alexander Ayriyan ${ }^{1, \star}$ and Hovik Grigorian ${ }^{1,2, \star \star}$ \\ ${ }^{1}$ Joint Institute for Nuclear Research, Joliot-Curie 6, 141980 Dubna, Russia \\ ${ }^{2}$ Yerevan State University, Alek Manyukyan 1, 0025 Yerevan, Republic of Armenia
}

\begin{abstract}
A simple mixed phase model mimicking so-called "pasta" phases in the quarkhadron phase transition is developed and applied to static neutron stars for the case of DD2 type hadronic and NJL type quark matter models. The influence of the mixed phase on the mass-radius relation of the compact stars is investigated. Model parameters are chosen such that the results are in agreement with the mass-radius constraints.
\end{abstract}

\section{Introduction and Motivation}

The possible hadron-quark phase transition modelling is usually made with the use of the so-called Maxwell construction, where the two phases are assumed to be separated. However, due to the finite surface tension effects, a mixed phase with non-trivial structure (the so-called "pasta" phase) could be thermodynamically preferred [1-6].

A simple model of phase transition construction mimicking the "pasta" phase is proposed. The model is parameterized by the use of an additional pressure corresponding to the impact of the structural effects in the mixed phase to the critical pressure of the Maxwell construction. The construction ignoring the details of the mixed phase structure is simple for calculations and therefore it can be easily used for investigations of the influence of the mixed phase on the mechanical characteristics of the compact stars [5].

The question of the existence of the mixed phase is actual in the investigations of the hybrid compact star structures and characteristics, which could be compared to the available observational data $[7,8]$. In the sequel an application of this model to the structure and mass-radius relation of the static neutron stars is presented using the hadronic matter model with density-dependent mesonnucleon coupling (DD2) [9], and the Nambu-Jona-Lasinio model (NJL) with 8-quark interaction [10] for quark matter. The method could be extended to the finite temperature case along the whole first oder transition border of the QCD phase diagram and could be used in the simulations of heavy ion collisions as well (e.g. [11, 12]). The possibility of such extension is also discussed.

\section{The model}

We assume that the hadronic and quark matter phases are described by equation of state (EoS) denoted $P_{H}(\mu)$ and $P_{Q}(\mu)$ respectively which describe the dependence of pressure on chemical potential at $T=0$ case relevant for the netrons star (NS) modeling.

\footnotetext{
^e-mail: ayriyan@jinr.ru

$\star \star$ e-mail: hovik.grigorian@gmail.com
} 
It is assumed that a first order Maxwell phase transition exists between the given two phases with a critical chemical potential $\mu_{c}$ satisfying the following relation:

$$
P_{Q}\left(\mu_{c}\right)=P_{H}\left(\mu_{c}\right)=P_{c} .
$$

The Maxwell construction can be modified if the assumption is fulfilled that close to the phase transition point the EoS of both phases are changing due to their interactions (see fig. 1), so that the effective mixed phase EoS can be described as

$$
P_{M}(\mu)=\alpha\left(\mu-\mu_{c}\right)^{p}+\beta\left(\mu-\mu_{c}\right)^{q}+P_{c}+\Delta P,
$$

where $p>q$ are natural numbers for instance. Here the additional pressure at $\mu_{c}$ is considered to be the free parameter of the model:

$$
P_{M}\left(\mu_{c}\right)=P_{H}\left(\mu_{c}\right)+\Delta P .
$$

This research is focused on the quadratic form of the EoS of the mixed phase ( $p=2$ and $q=1$ ):

$$
P_{M}(\mu)=\alpha\left(\mu-\mu_{c}\right)^{2}+\beta\left(\mu-\mu_{c}\right)+P_{c}+\Delta P .
$$

The transition from the $H$-phase to the $M$-phase is smooth without jump in the density $n(\mu)=$ $d P(\mu) / d \mu$. Thus there are the new critical chemical potentials: $\mu_{c H}$ for the transition from the $H$-phase to the $M$-phase and $\mu_{c Q}$ for the transition from the $M$-phase to the $Q$-phase. As a result, there are four unknowns including the coefficients $\alpha$ and $\beta$ from Eq. (2). The phase transition conditions are

$$
\left\{\begin{array} { l } 
{ P _ { M } ( \mu _ { c H } ) = P _ { H } ( \mu _ { c H } ) , } \\
{ P _ { M } ( \mu _ { c Q } ) = P _ { Q } ( \mu _ { c Q } ) , }
\end{array} \quad \left\{\begin{array}{l}
n_{M}\left(\mu_{c H}\right)=n_{H}\left(\mu_{c H}\right), \\
n_{M}\left(\mu_{c Q}\right)=n_{Q}\left(\mu_{c Q}\right) .
\end{array}\right.\right.
$$

The quantities $\alpha$ and $\beta$ are expressed from the set of equations for the pressure

$$
\left\{\begin{array}{l}
\alpha=\frac{1}{\mu_{c Q}-\mu_{c H}}\left(\frac{P_{Q}\left(\mu_{c Q}\right)-\left(\Delta P+P_{c}\right)}{\mu_{c Q}-\mu_{c}}-\frac{P_{H}\left(\mu_{c H}\right)-\left(\Delta P+P_{c}\right)}{\mu_{c H}-\mu_{c}}\right), \\
\beta=\frac{P_{H}\left(\mu_{c H}\right)-\left(\Delta P+P_{c}\right)}{\mu_{c H}-\mu_{c}}+\frac{P_{Q}\left(\mu_{c Q}\right)-\left(\Delta P+P_{c}\right)}{\mu_{c Q}-\mu_{c}}-\frac{P_{Q}\left(\mu_{c Q}\right)-P_{H}\left(\mu_{c H}\right)}{\mu_{c Q}-\mu_{c H}},
\end{array}\right.
$$

and are eliminated form the set of equations for the densities (eq. (5)). Solving the remaining equations for the densities one can find the values of the critical chemical potentials $\mu_{c H}$ and $\mu_{c Q}$.

\section{Results}

For the illustration purposes, the described model is applied for the construction the mixed phase EoS (see fig. 1) between the hadronic and quark matter respectively given by the DD2 type model [9] and the NJL model with 8-quark interaction [10]. The results of the construction are shown in fig. 2. Here the varied pressure is given by the dimensionless parameter $\Delta_{P}=\Delta P / P_{c}$. The parameter $\Delta_{P}$ is varied within $10 \%$. This completely covers the whole range of the additional pressure obtained by the microscopic models of the mixed phase $[1-4,6]$. Note, that the $P-\varepsilon$ dependence of the mixed phase is not a linear function by the construction, but is very close to it.

With the use of this set of the EoS models the mechanical characteristics of the compact stars are investigated. The mass and radius relationships of the static spherically symmetric neutron stars are 


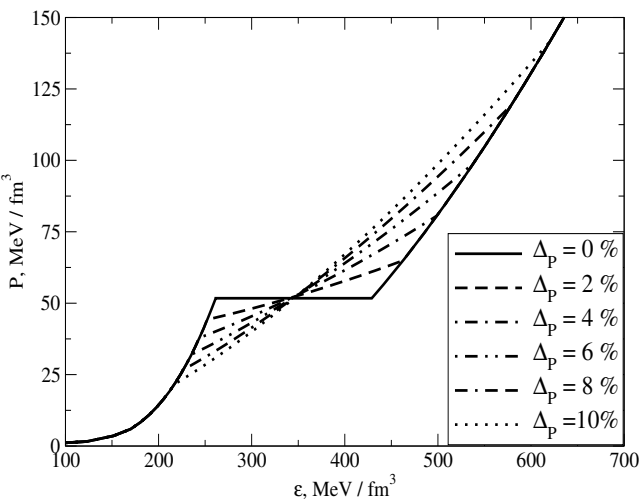

Figure 2. Influence of the additional pressure in the mixed phase on the phase transition.

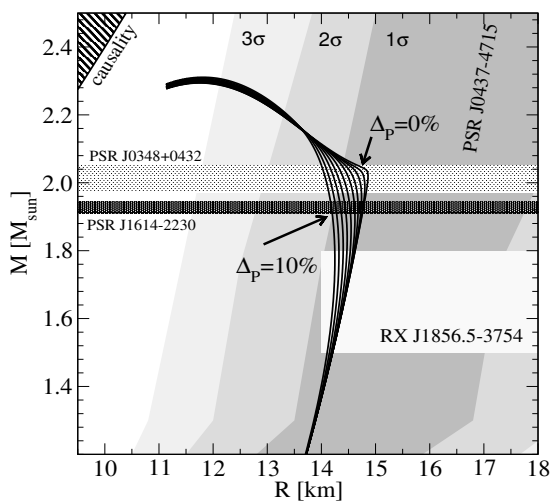

Figure 3. Influence of the mixed phase on the mass-radius relation.

obtained as solutions of the Tolman-Oppenheimer-Volkoff (TOV) equations [13, 14]:

$$
\left\{\begin{array}{l}
\frac{d P(r)}{d r}=-\frac{G M(r) \varepsilon(r)}{r^{2}} \frac{\left(1+\frac{P(r)}{\varepsilon(r)}\right)\left(1+\frac{4 \pi r^{3} P(r)}{M(r)}\right)}{\left(1-\frac{2 G M(r)}{r}\right)}, \\
\frac{d M(r)}{d r}=4 \pi r^{2} \varepsilon(r),
\end{array}\right.
$$

where $\hbar=c=1, r$ is the coordinate distance from the center, $M(r), \varepsilon(r)$ and $P(r)$ are the mass, the energy density and the pressure profiles correspondingly, and $G$ is the gravitational constant. Each of these profiles, representing solutions of the TOV equations, depends on the value of the energy density in the star center $\varepsilon(0)$. The relation between the radius $R$ where the pressure is $P(R)=0$ and the total mas $M=M(R)$ are shown in the fig. 3 for different values of the parameter $\Delta_{P}$.

The presence of the mixed phase changes the EoS softness and the maximum mass becomes larger when the maximum radius decreases. The heaviest known pulsars PSR J0348+0432 and PSR J1614-2230 have respectively the following masses $2.01_{-0.04}^{+0.04} \mathrm{M}_{\odot}$ [15] and $1.928_{-0.017}^{+0.017} \mathrm{M}_{\odot}[16$, 17]. Therefore the maximum possible mass given by a model should exceed this limits. These constraints are shown in the fig. 3. It is demonstrated that all considered EoS models with the mixed phase satisfy this requirement. On this plot the constraints from the radius estimations of the two pulsars PSR J0437-4715 [18] and RX J1856.5-3754 [19] are also shown and the results are in agreement with this.

\section{Conclusions and Discussion}

A simple model of the hadron-quark phase transition mimicking the "pasta" structure is proposed. It is parameterized using the additional pressure due to the structure of the mixed phase. The model is based on the standard Maxwell construction of the first order phase transitions. It ignores the details of the mixed phase structure, at the same time it is easy to use for the investigation of the influence of the mixed phase on the mechanical characteristics of the compact star. The structure and mass-radius relations of the static neutron stars are presented for the case of the DD2 type hadronic vs NJL type quark matter EoS models. It is shown that the variation of the parameter $\Delta_{P}$ in the relevant range of the additional pressure, for the mixed phase, changes the maximum mass and the radius of the star configurations within limits imposed by the observational constraints. 
The method could be extended to the finite temperature case along the whole first oder transition border of the QCD phase diagram as it is shown in the fig.4 assuming that the additional pressure decreases roportionally to the increases in the temperature. Thus the case when the additional pressure goes from the value $\Delta_{P}$ at $\left(\mu=\mu_{c}, T=0\right)$ down to zero at the Critical End Point (CEP) is demonstrated in fig. 4. The first order phase transition (the jump in density) disappears at the $\mathrm{CEP}$, therefore the possible mixed phase is also disappearing. For the crossover transition one needs to apply another construction. The considered approach for the transition construction is independent of the internal details of the transition, therefore it could be applied both for the beta-equilibrated and for the symmetric nuclear matter. Thus it could be used in the simulations of heavy ion collisions.

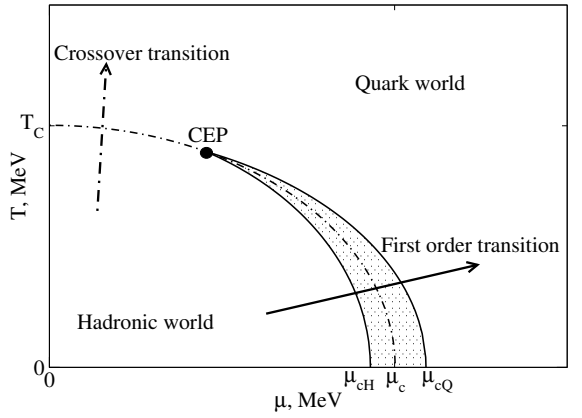

Figure 4. A simple schematic view to the QCD phase diagram. The grey area is the area of mixed phase of the first order hadron-quark phase transition.

\section{Acknowledgements}

The authors thank Prof. David Blaschke, Prof. Dmitry Voskresensky, Dr. David Edwin Alvarez Castillo, Dr. Vahagn Abgaryan and Konstantin Maslov for fruitful discussions. Prof. Stefan Typel and Dr. Sanjin Benic provided tables of the EoS models, special thanks to them. The research was carried out under financial support of the Russian Science Foundation (project No. 17-12-01427).

\section{References}

[1] D. N. Voskresensky et al., Nucl. Phys. A 723, 291-339 (2003)

[2] T. Tatsumi et al., Nucl. Phys. A 718, 359-362 (2003)

[3] T. Endo et al., Nucl. Phys. A 749, 333-336 (2005)

[4] T. Endo et al., Prog. Theor. Phys. 115 (2), 337-353 (2006)

[5] T. Noda et al., Astrophys. J. 765:1 (2013)

[6] N. Yasutake et al., Phys. Rev. C 89, 065803 (2014)

[7] M. Bejger, P. Haensel, and J. L. Zdunik, Mon. Not. Roy. Astron. Soc. 359, 699-706 (2005)

[8] D. E. Alvarez-Castillo and D. Blaschke, Phys. Part. Nucl. 46, 846-848 (2015)

[9] S. Typel, Eur. Phys. J. A 52:16 (2016)

[10] S. Benic, Eur. Phys. J. A 50:111 (2014)

[11] V. D. Toneev, Eur. Phys. J. WoC 7, 02005 (2010)

[12] K. A. Bugaev et al., Phys. Part. Nucl. Lett. 12 (2), 238-245 (2015)

[13] R. C. Tolman, Phys. Rev. 55 (4), 364-373 (1939)

[14] J. R. Oppenheimer and G. M. Volkoff, Phys. Rev. 55 (4), 374-381 (1939)

[15] J. Antoniadis, P. C. C. Freire, N. Wex, et al., Science 340, 6131 (2013)

[16] P. Demorest, T. Pennucci, S. Ransom, et al., Nature 467, 1081-1083 (2010)

[17] E. Fonseca et al., Astrophys. J. 832, 167 (2016).

[18] S. Bogdanov, Astrophys. J. 762:96 (2013)

[19] H. Hambaryan, R. Neuhauser, V. Suleimanov, and K. Werner, J. Phys., Conf. Ser. 496, 012015 (2014) 